\title{
Yellow Emissive Tris(8-hydroxyquinoline) Aluminum by the Incorporation of ZnO Quantum Dots for OLED Applications
}

\author{
Aya Hekmet Makki ${ }^{1,2}$ and Si-Hyun Park ${ }^{1, *(D)}$ \\ 1 Department of Electronics Engineering, Yeungnam University, Gyeongsan 38541, Korea; ayah@ynu.ac.kr \\ 2 Laser and Optoelectronics Engineering Department, University of Technology-Iraq, Baghdad 10066, Iraq \\ * Correspondence: sihyun_park@ynu.ac.kr
}

Citation: Makki, A.H.; Park, S.-H. Yellow Emissive Tris(8-hydroxyquinoline) Aluminum by the Incorporation of ZnO Quantum Dots for OLED Applications. Micromachines 2021, 12, 1173. https://doi.org/10.3390/ mi12101173

Academic Editor:

Takafumi Fukushima

Received: 9 September 2021

Accepted: 27 September 2021

Published: 29 September 2021

Publisher's Note: MDPI stays neutral with regard to jurisdictional claims in published maps and institutional affiliations.

Copyright: (c) 2021 by the authors. Licensee MDPI, Basel, Switzerland. This article is an open access article distributed under the terms and conditions of the Creative Commons Attribution (CC BY) license (https:/ / creativecommons.org/licenses/by/ $4.0 /)$.

\begin{abstract}
Tris(8-hydroxyquinoline) aluminum complexes are of significant interest because of their remarkable optical and electrical properties, both as an emissive layer and electron injection layer. They emit light in the blue and green ranges of the visible spectrum, so for white organic light emitting diodes (OLEDs), yellow emission is required as well. In this study, we propose the use of zinc oxide quantum dots to tune the emission color of the complex while maintaining its luminous efficiency. Hence, tris(8-hydroxyquinoline) aluminum-zinc oxide nanohybrids with different zinc oxide quantum dots concentrations $(10,20$, or $30 \mathrm{wt}$. $\%$ ) were synthesized. The structural properties were characterized using powder X-ray diffraction analysis, while the composition and optical characteristics were characterized by Fourier transform infrared spectroscopy, UV-visible absorption spectroscopy, and photoluminescence emission spectroscopy. The results show that increased levels of zinc oxide quantum dots lead to a decrease in crystallinity, double hump emission and a slight red shift in emission peaks. Also, at 20 and $30 \mathrm{wt}$.\% of zinc oxide quantum dots concentrations, yellow emission was observed.
\end{abstract}

Keywords: OLED; Alq3; Alq3-ZnO nanohybrids

\section{Introduction}

Organic light-emitting diodes (OLEDs) have attracted significant research attention, especially for next-generation flexible and foldable devices compared with their inorganic counterparts, owing to their outstanding material properties, such as high flexibility, uniform emission over a large area, tunable wavelength, ease of fabrication, low cost and power consumption, and environmental friendliness [1-5]. Throughout the last couple of years, the study has therefore focused on organic-electroluminescent materials. Among those materials, Tris (8-hydroxyquinoline) aluminum (III) (Alq3) has been attracted and seen as a promising candidate for its excellent electrical transport and emission properties, as well as its high thermal stability. As a result, it is a better choice for emissive and electrontransparent layers in OLED and organic light-emitting transistor (OLET) devices [6-8].

Alq3 is an organometallic semiconductor material which exists in several crystalline phases $(\alpha, \beta, \gamma, \delta$, and $\varepsilon)$ depending on the synthesis method. Previous studies have shown blue fluorescence in $\delta$ - and $\gamma$ phases while green fluorescence was found in the other phases under UV excitation [9-11].

Color tuning in Alq3 was achieved previously by chemical means in which the band gap, and therefore complex emission wavelength, was tailored by the attachment of electron-donating or electron withdrawing substituents to the quinolinolate ligand [12,13].

The only drawback to Alq3 complexes is their high environmental sensitivity and ease of photooxidation, which results in low stability and hence luminescence efficiency degradation, subsequently limiting their use in OLED devices [14]. Many researchers have proposed the use of hybrid (organometallic/inorganic) composites to overcome these issues. Besides, those hybrids showed significant improvements in mechanical strength, thermal 
stability, luminescent efficiency, and charge mobility when compared to pure Alq3 [15,16]. Cuba et al. [17] showed that Alq3-ZnO composites exhibited enhanced luminescence properties and a slight shift from green emission to greenish blue. Li et al. [18]. reported that Alq3-nano-TiO2 using 8-vinyl POSS as a modifier resulted in an enhancement in the photoluminescence (PL) and electroluminescence (EL) properties of the composites. In this study, we report the yellow emission of Alq3 upon the introduction of $\mathrm{ZnO}$ quantum dots (QDs) as a dopant and studied the effect of $\mathrm{ZnO}$ QDs concentration on the structural and optical properties of the samples. Alq3- $\mathrm{ZnO}$ nanohybrids were synthesized using varying concentrations of $\mathrm{ZnO}$ QDs (10, 20, and $30 \mathrm{wt} . \%)$.

\section{Materials and Methods}

\subsection{Materials}

Multi-wall carbon nanotubes (MWCNTs, 98\%, Sigma Aldrich, Seoul, Korea), zinc acetate dihydrate $\left(\mathrm{Zn}(\mathrm{OAc})_{2} \cdot 2 \mathrm{H}_{2} \mathrm{O}, 98 \%\right.$, Duksan, Seoul, Korea), hydrochloric acid $(\mathrm{HCl}$, $35 \%$, Duksan, Seoul, Korea), Sulfuric acid $\left(\mathrm{H}_{2} \mathrm{SO}_{4}, 98 \%\right.$, Duksan, Seoul, Korea), ethanol $\left(\mathrm{CH}_{3} \mathrm{CH}_{2} \mathrm{OH}, 99.9 \%\right.$, Duksan, Seoul, Korea), 8-hydroxyquinoline (99\%, Junsei Chemical, Tokyo, Japan), aluminum chloride $\left(\mathrm{AlCl}_{3}, 99 \%\right.$, Fluka, Seoul, Korea), and potassium hydroxide (KOH, 85\%, DAEJUNG, Seoul, Korea). All chemicals were used as received without any further purification.

\subsection{Methods}

\subsubsection{Synthesis of $\mathrm{ZnO}$ QDs}

Yellow emissive $\mathrm{ZnO}$ QDs were synthesized according to the procedure described by Yang et al. [19]. Multi-walled carbon nanotubes (MWCNTs) were functionalized with acidic treatment to produce functional MWCNTs (FMWCNTs). A $20 \mathrm{mg}$ sample of FMWCNTs were suspended in ethanol $(20 \mathrm{~mL})$ with sonication for $20 \mathrm{~min}$. A $6 \mathrm{~mL}$ aliquot was taken from suspended FMWCNT solution and added to an ethanolic zinc acetate dihydrate solution $(0.09 \mathrm{M})$ and stirred at room temp for $30 \mathrm{~min}$, followed by refluxing at $70{ }^{\circ} \mathrm{C}$ for $4 \mathrm{~h}$. The solution was subsequently cooled and sonicated for $30 \mathrm{~min}$, and then the supernatant containing suspended ZnO QDs was collected. The QDs were collected by centrifugation, cleaned several times with ethanol and water, and dried in an oven at $60^{\circ}$ at atmospheric pressure.

\subsubsection{Synthesize of Alq3}

Alq3 was synthesized as follows: $0.3 \mathrm{M}$ of ethanolic 8-hydroxyquinoline solution was mixed with a $0.22 \mathrm{M} \mathrm{AlCl}_{3}$ ethanolic solution and the $\mathrm{pH}$ was neutralized using a $\mathrm{KOH}$ solution. The resulting mixture was refluxed at $70{ }^{\circ} \mathrm{C}$ for $5 \mathrm{~h}$ with stirring. The reaction mixture was subsequently allowed to cool to room temperature, and the yellow Alq3 precipitate was collected by centrifugation, washed with ethanol, and deionized water, and dried under vacuum at $100{ }^{\circ} \mathrm{C}$ for $12 \mathrm{~h}$.

\subsubsection{Synthesis of Alq3-ZnO Nanohybrids}

To synthesize Alq3-10 wt.\% ZnO QDs, Alq3 (180 mg) was suspended in ethanol (32.5 mL) with stirring. ZnO QDs (20 mg) were also suspended in ethanol $(3.25 \mathrm{~mL})$ and sonicated. After $15 \mathrm{~min}$, both suspensions were added with stirring and heated to $60^{\circ} \mathrm{C}$ for $4 \mathrm{hrs}$. A precipitate was formed upon cooling which was collected by centrifugation and decantation, washed with ethanol, deionized water and dried under vacuum at $100{ }^{\circ} \mathrm{C}$ for $12 \mathrm{~h}$. Identical procedures were used for the synthesis of Alq3-20 wt.\% ZnO QD and Alq330 wt.\% ZnO QD. For characterization, the MPD (Panalytical, Malvern, United Kingdom) Xpert Multipurpose X-ray Diffraction System was used for powder X-ray diffraction (XRD) analysis. A Spectrum 100 spectrometer (Perkin-Elmer, Waltham, Massachusetts, United States) and a Cary 5000 UV-vis-NIR spectrophotometer (Agilent Technologies, Santa Clara, California, United States) were used to record the Fourier transform infrared (FTIR) and absorption spectra, respectively. 


\section{Results and Discussion}

Figure 1 illustrates the structural properties of the synthesized Alq3, ZnO QDs, and Alq3-ZnO nanohybrids studied by XRD. The distinctive peaks of Alq3 and hexagonal $\mathrm{ZnO}$ observed in the XRD spectra are in agreement with the stated values standard (JCPDS 26-1550) and (JCPDS 75-0576), respectively. The crystallite sizes of the ZnO QDs were estimated using the Debye-Sherrer equation [20] and found to be 8.78, 8.85, 10.67, 11.08, 9.6, 10.41 , and $10.04 \mathrm{~nm}$ for the (100), (002), (101), (102), (110), (103), and (112) crystalline phases, respectively. Figure $1 \mathrm{c}$ shows the diffraction pattern of Alq3-ZnO nanohybrids at different concentration levels of $\mathrm{ZnO}$ QDs. Increasing in $\mathrm{ZnO}$ QDs concentration results in a slight decrease in peaks intensity as well as a slight shifting of the diffraction peaks toward lower diffraction angles which confirms the incorporation of the $\mathrm{ZnO}$ QDs in the Alq3 matrix, resulting in positional rearrangement in the Alq3 lattice. In addition, no individual $\mathrm{ZnO}$ QDs peaks were observed for all the samples.
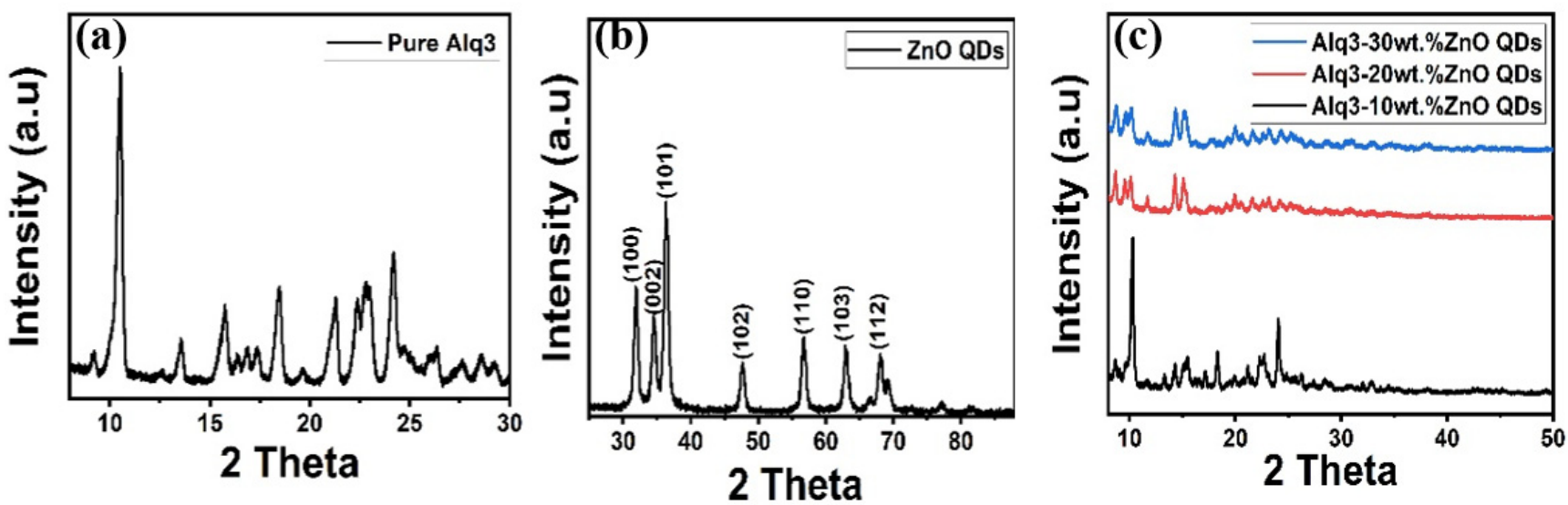

Figure 1. XRD pattern of (a) pure Alq3, (b) ZnO QDs and (c) Alq3-ZnO nanohybrids.

The composition of the Alq3 and Alq3-ZnO QD nanohybrids was studied by FTIR spectroscopy as shown in Figure 2. The FTIR spectrum of Alq3 shows all the expected characteristic peaks, as shown in Table 1. Doping with ZnO QDs resulted in weakening of the absorbance band in the $400-600 \mathrm{~cm}^{-1}$ region and shifting of several absorbance peaks. These observations are an indication of the interactions between the $\mathrm{ZnO}$ QDs and Alq3 and are consistent with the results of the XRD analysis. Notably, an absorption band around $500 \mathrm{~cm}^{-1}$ broadened with increasing $\mathrm{ZnO}$ QD concentration, which was attributed to $\mathrm{Zn}-\mathrm{O}$ vibrations.

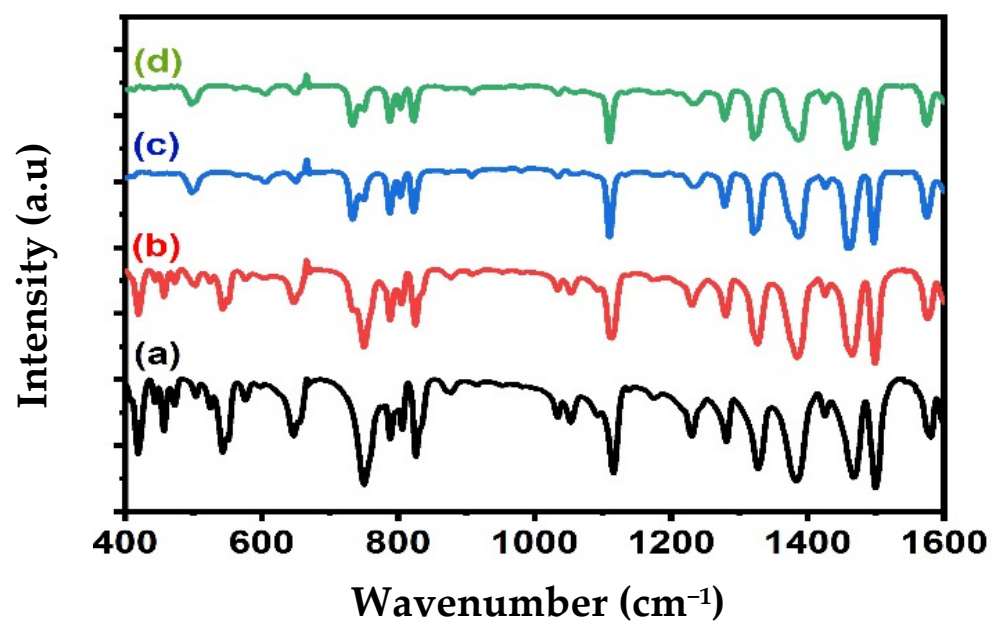

Figure 2. FTIR spectrum of (a) pure Alq3, (b) Alq3-10 wt.\% ZnO QDs (c) Alq3-20 wt.\% ZnO QDs (d) Alq3-30 wt. $\%$ ZnO QDs. 
Table 1. IR Vibrational modes in FTIR spectra of pure Alq3 and Alq3-ZnO nanohybrids [3].

\begin{tabular}{|c|c|c|c|c|c|c|c|c|c|}
\hline \multicolumn{4}{|c|}{ Wavenumber $\left(\mathrm{cm}^{-1}\right)$} & \multicolumn{6}{|c|}{ Wavenumber $\left(\mathrm{cm}^{-1}\right)$} \\
\hline Alq3 & $\begin{array}{c}\text { Alq3-10 } \\
\text { wt. } \%\end{array}$ & $\begin{array}{c}\text { Alq3-20 } \\
\text { wt. } \%\end{array}$ & $\begin{array}{c}\text { Alq3-30 } \\
\text { wt. } \%\end{array}$ & Assignment & Alq3 & $\begin{array}{c}\text { Alq3-10 } \\
\text { wt. } \%\end{array}$ & $\begin{array}{c}\text { Alq3-20 } \\
\text { wt. } \%\end{array}$ & $\begin{array}{c}\text { Alq3-30 } \\
\text { wt. } \%\end{array}$ & Assignment \\
\hline 419 & 419 & 420 & 422 & Al-N vibration & 1053 & 1053 & 1056 & 1056 & C-O stretching \\
\hline 444 & 444 & 444 & 445 & Al-O stretching & 1115 & 1113 & 1110 & 1110 & C-O stretching \\
\hline 457 & 457 & 459 & 459 & Al-O stretching & 1138 & 1137 & 1133 & 1133 & C-O stretching \\
\hline 473 & 473 & 473 & 473 & Al-O stretching & 1175 & 1173 & 1173 & 1173 & C-O stretching \\
\hline 504 & 503 & 497 & 497 & Al-O stretching & 1231 & 1231 & 1236 & 1232 & C-O stretching \\
\hline 543 & 543 & 545 & 545 & Al-O stretching & 1281 & 1280 & 1278 & 1278 & Aromatic amine \\
\hline 576 & 576 & 576 & 576 & $\mathrm{C}-\mathrm{H}$ bending & 1327 & 1327 & 1321 & 1321 & Aromatic amine \\
\hline 599 & 601 & 605 & 606 & $\mathrm{C}-\mathrm{H}$ bending & 1383 & 1384 & 1386 & 1278 & Aromatic amine \\
\hline 647 & 648 & 651 & 651 & $\mathrm{C}-\mathrm{H}$ bending & 1425 & 1425 & 1426 & 1321 & $\mathrm{C}=\mathrm{C}$ stretching \\
\hline 750 & 750 & 733 & 733 & $\mathrm{C}-\mathrm{H}$ bending & 1468 & 1466 & 1460 & 1385 & $\mathrm{C}=\mathrm{C}$ stretching \\
\hline 788 & 788 & 788 & 187 & C-H bending & 1499 & 1499 & 1497 & 1497 & $\mathrm{C}=\mathrm{C}$ bending \\
\hline 806 & 805 & 803 & 803 & $\mathrm{C}-\mathrm{H}$ bending & 1580 & 1576 & 1575 & 1575 & $\mathrm{C}=\mathrm{C}$ bending \\
\hline 877 & 877 & 823 & 823 & $\mathrm{C}-\mathrm{H}$ bending & 1605 & 1604 & 1603 & 1603 & $\mathrm{C}=\mathrm{C}$ bending \\
\hline 1034 & 1034 & 1034 & 1034 & C-O stretching & & & & & \\
\hline
\end{tabular}

The absorption spectra of Alq3 and Alq3-ZnO nanohybrids are shown in Figure 3. The spectrum of Alq3 showed an absorption peak at approximately $400 \mathrm{~nm}$ corresponding to the $\pi-\pi^{*}$ electronic transitions of the quinolinolate ligands [17]. Doping with $\mathrm{ZnO}$ QDs resulted in the emergence of an additional peak at approximately $350 \mathrm{~nm}$ which corresponded to the direct band gap transition of ZnO QDs [21]. The band gap energy of the samples was estimated using the Tauc method [22] with the following equation:

$$
\alpha h v=(h v-E g)^{n},
$$

where $n$ is $1 / 2$ for allowed direct transitions, and 2 for allowed indirect transitions [22]. The direct band gap was estimated to be $2.79 \mathrm{eV}$ for Alq3, which is consistent with the previously reported value [22]. The addition of $\mathrm{ZnO}$ QDs to Alq3 results in a slight decrease in the band gap with increasing concentration. The calculated bandgap energies of the Alq3-ZnO nanocomposites were calculated to be 2.76, 2.73, and $2.72 \mathrm{eV}$ for Alq3-10 wt.\% ZnO QDs, Alq3-20 wt.\% ZnO QDs, and Alq3-10 wt.\% ZnO QDs, respectively (Figure 4).

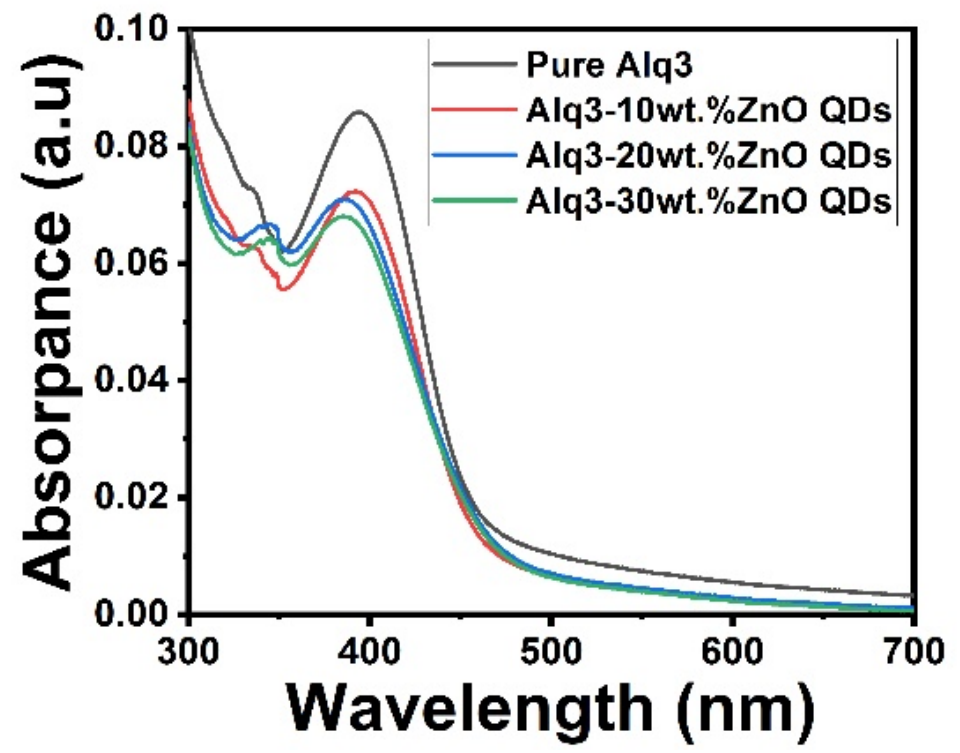

Figure 3. UV-Vis absorption spectrum of pure Alq3 and Alq3-ZnO nanohybrids. 

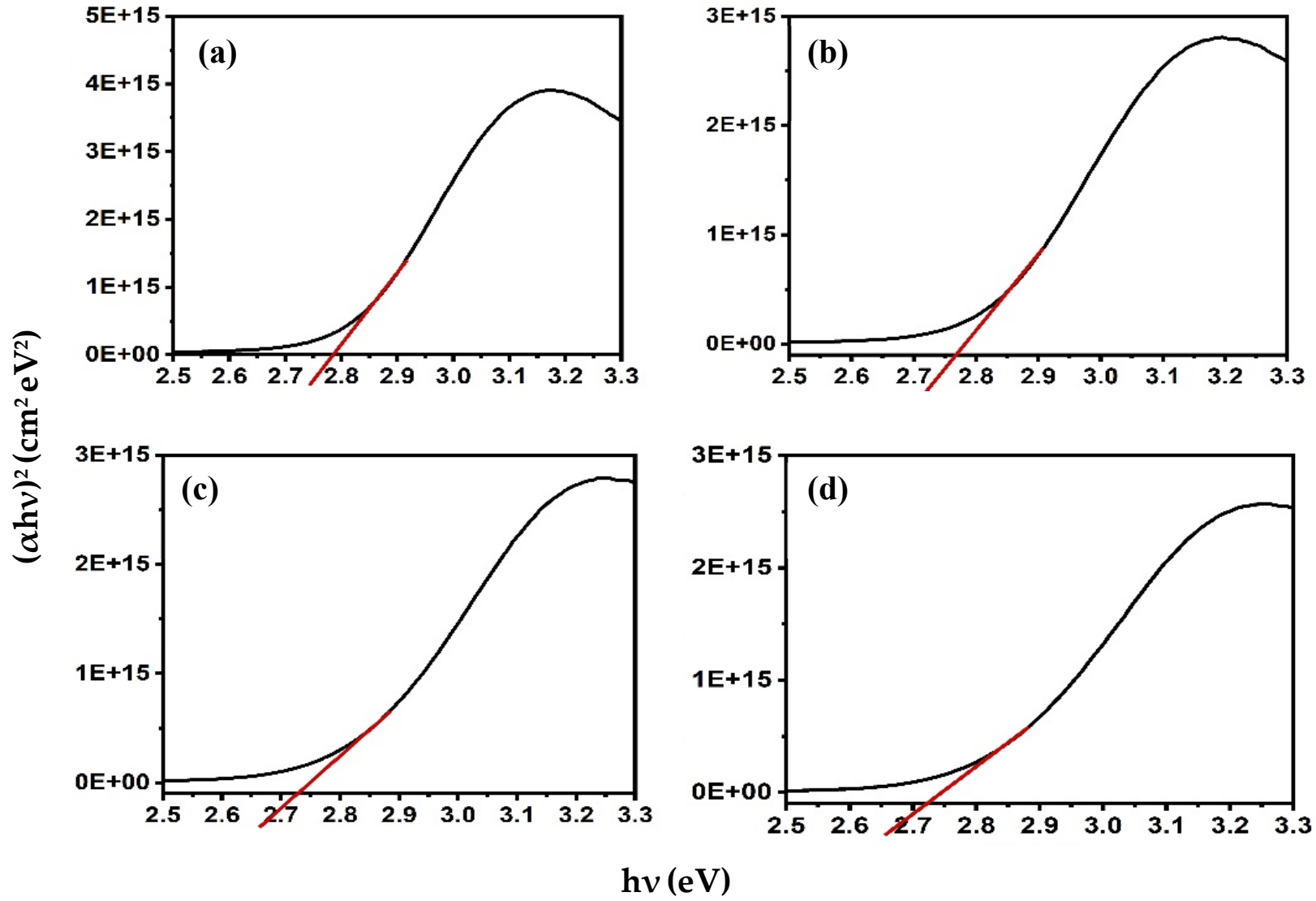

Figure 4. $(\alpha \mathrm{h} v)^{2}$ versus hv curves for (a) pure Alq3, (b) Alq3-10 wt.\% ZnO QDs (c) Alq3-20 wt.\% ZnO QDs (d) Alq3-30 wt.\% ZnO QDs.

The PL spectra of Alq3 and Alq3-ZnO nanohybrids after excitation with a He-Cd laser $(325 \mathrm{~nm})$ are shown in Figure 5. Pure Alq3 exhibits green emission centered around $525 \mathrm{~nm}$. The presence of $\mathrm{ZnO}$ QDs results in introducing a new energy sate within the energy band gap of the Alq3 and hence results in a double-hump emission, the first due to the Alq3 molecules and the second due to the presence of ZnO QDs. Additionally, the emission peaks gradually red shifted with increasing $\mathrm{ZnO}$ QD concentration. The integrated PL intensity was found to be almost identical for all samples. The deconvoluted emission spectra are shown in Figure 6. The emission hump at approximately $520 \mathrm{~nm}$ correspond to Alq3, while the emission hump at approximately $560 \mathrm{~nm}$ corresponds to the $\mathrm{ZnO}$ QDs.
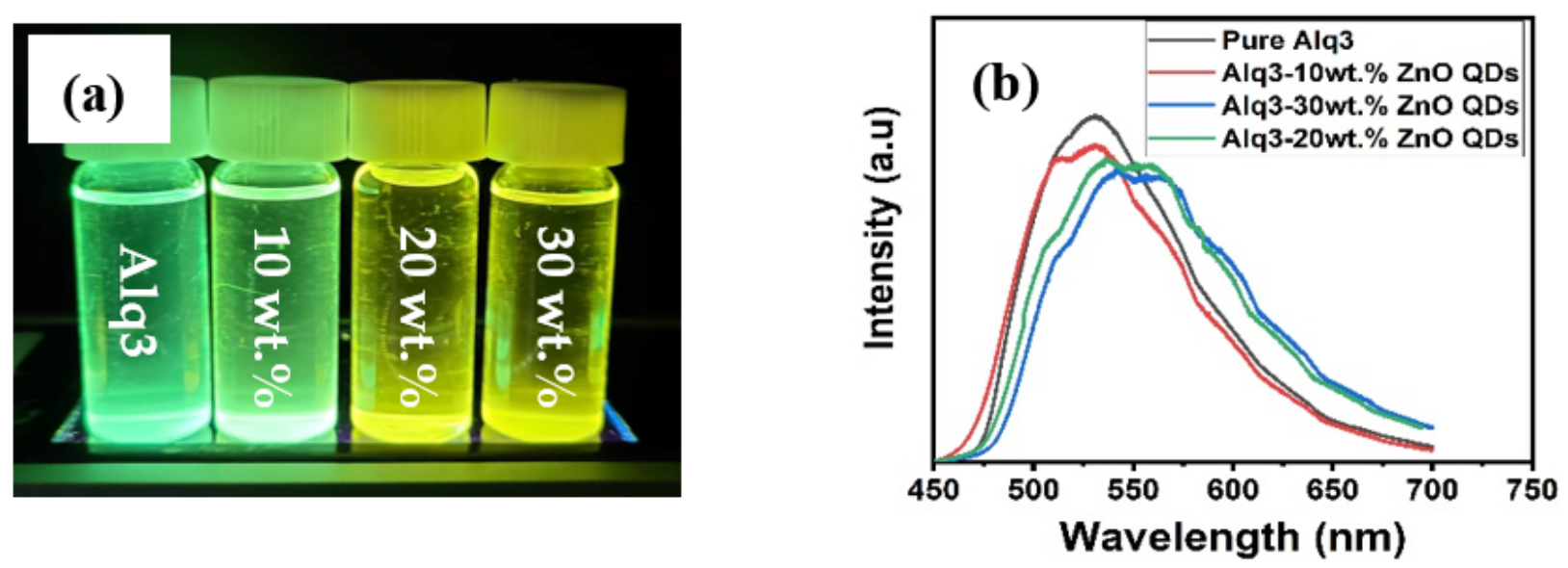

Figure 5. (a) Flurecence of pure Alq3 and Alq3-ZnO nanohybrids under UV excitation (b) PL emission spectrum. 

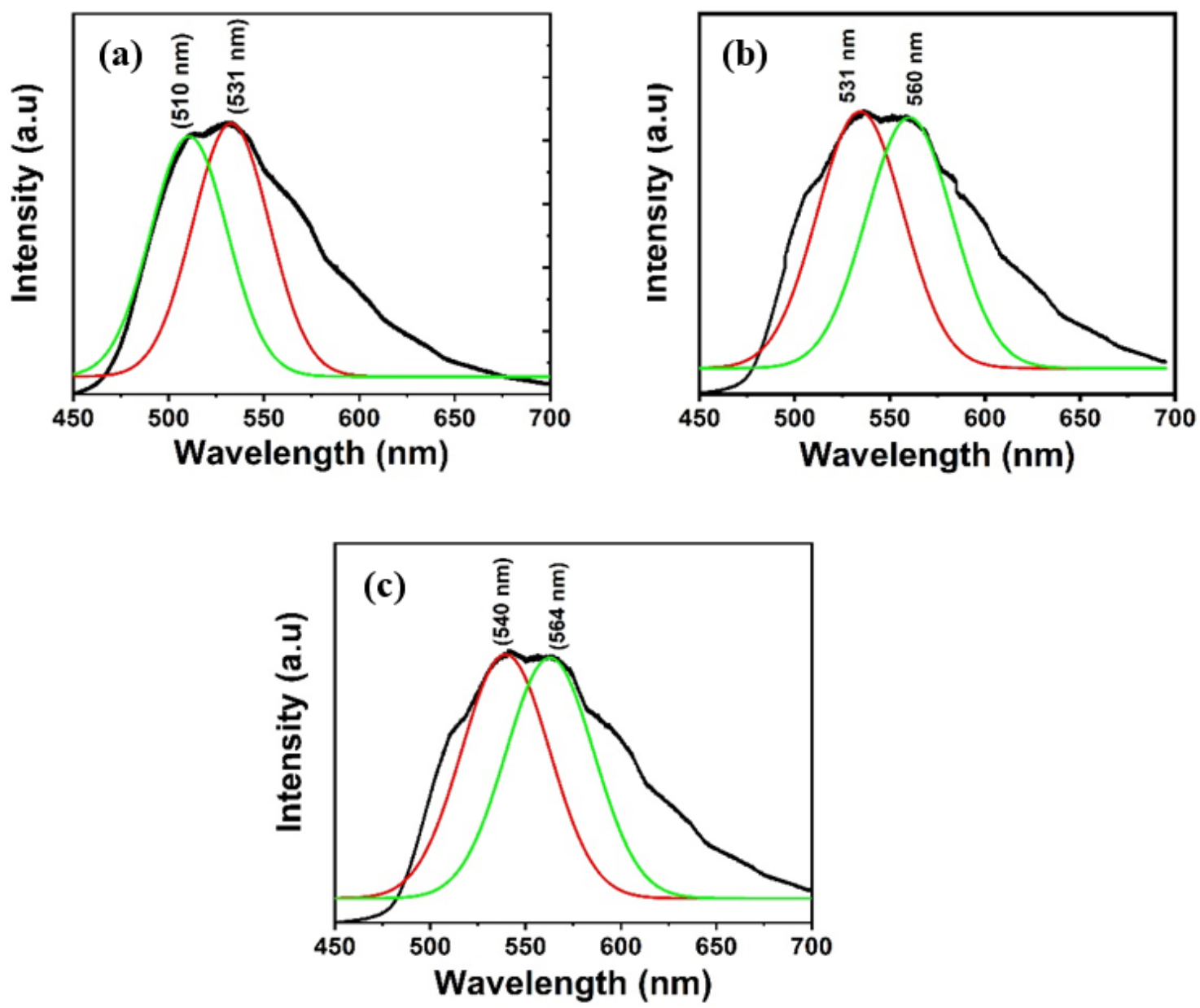

Figure 6. Deconvolution of the emission spectra of Alq3-ZnO nanohybrids. (a) Alq3-10 wt.\% ZnO QDs (b) Alq3-20 wt.\% ZnO QDs (c) Alq3-30 wt.\% ZnO QDs.

\section{Conclusions}

A yellow emissive Alq3 is obtained through physical means by doping with $\mathrm{ZnO}$ QDs. The Alq3-ZnO nanohybrids containing 10, 20, and $30 \mathrm{wt} . \% \mathrm{ZnO}$ QDs were synthesized. Pure Alq3 exhibits green emission at $530 \mathrm{~nm}$ under excitation at $325 \mathrm{~nm}$ while doping with $\mathrm{ZnO}$ QDs results in a yellow emission peak centered at $560 \mathrm{~nm}$ for Alq3-20 wt.\% ZnO QDs and Alq3-30 wt.\% ZnO QDs. Therefore, based on our findings the color tuning in Alq3 can be achieved using ZnO QDs while maintaining the intrinsic PL intensity. This study demonstrates that these nanohybrids can be considered as promising candidates for use as emitting and transparent layers in yellow and white emissive OLEDs and OLETs.

Author Contributions: Conceptualization, A.H.M. and S.-H.P.; methodology, A.H.M.; software, A.H.M.; validation, A.H.M. and S.-H.P.; formal analysis, A.H.M.; investigation, A.H.M.; resources, A.H.M. and S.-H.P.; data curation, A.H.M.; writing-original draft preparation, A.H.M.; writingreview and editing, A.H.M. and S.-H.P.; visualization, S.-H.P.; supervision, S.-H.P.; project administration, S.-H.P.; funding acquisition, S.-H.P. All authors have read and agreed to the published version of the manuscript.

Funding: This work was supported by the 2021 Yeungnam University Research Grant.

Institutional Review Board Statement: Not applicable.

Informed Consent Statement: Not applicable.

Data Availability Statement: The data used to support the findings of this study are included within the article. 
Conflicts of Interest: The authors declare no conflict of interest.

\section{References}

1. Jeong, E.G.; Kwon, J.H.; Kang, K.S.; Jeong, S.Y.; Choi, K.C. A review of highly reliable flexible encapsulation technologies towards rollable and foldable OLEDs. J. Inf. Disp. 2020, 21, 19-32. [CrossRef]

2. Park, J.; Heo, S.; Park, K.; Song, M.H.; Kim, J.Y.; Kyung, G.; Ruoff, R.S.; Park, J.-U.; Bien, F. Research on flexible display at Ulsan National Institute of Science and Technology. NPJ Flex. Electron. 2017, 1, 9. [CrossRef]

3. Khan, M.B.; Khan, Z.H. Ag-incorporated Alq3 nanowires: Promising material for organic luminescent devices. J. Lumin. 2017, 188, 418-422. [CrossRef]

4. Zou, S.J.; Shen, Y.; Xie, F.M.; Chen, J.D.; Li, Y.Q.; Tang, J.X. Recent advances in organic light-emitting diodes: Toward smart lighting and displays. Mater. Chem. Front. 2020, 4, 788-820. [CrossRef]

5. Will, P.-A.; Reineke, S. 21-Organic light-emitting diodes. In Handbook of Organic Materials for Electronic and Photonic Devices, 2nd ed.; Ostroverkhova, O., Ed.; Woodhead Publishing: Sawston, UK, 2019; pp. 695-726.

6. El-Nahass, M.M.; Farid, A.M.; Atta, A.A. Structural and optical properties of Tris(8-hydroxyquinoline) aluminum (III) (Alq3) thermal evaporated thin films. J. Alloys Compd. 2010, 507, 112-119. [CrossRef]

7. Zawadzka, A.; Płóciennik, P.; Strzelecki, J.; Łukasiak, Z.; Sahraoui, B. Photophysical properties of Alq3 thin films. Opt. Mater. 2013, 36, 91-97. [CrossRef]

8. Cui, S.; Hu, Y.; Lou, Z.; Yi, R.; Hou, Y.; Teng, F. Light emitting field-effect transistors with vertical heterojunctions based on pentacene and tris-(8-hydroxyquinolinato) aluminum. Org. Electron. 2015, 22, 51-55. [CrossRef]

9. Rajeswaran, M.; Blanton, T.N.; Tang, C.W.; Lenhart, W.C.; Switalski, S.C.; Giesen, D.J.; Antalek, B.J.; Pawlik, T.D.; Kondakov, D.Y.; Zumbulyadis, N.; et al. Structural, thermal, and spectral characterization of the different crystalline forms of Alq3, tris(quinolin-8-olato)aluminum(III), an electroluminescent material in OLED technology. Polyhedron 2009, 28, 835-843. [CrossRef]

10. Painuly, D.; Mogha, N.K.; Masram, D.T.; Singhal, R.; Gedam, R.S.; Nagpure, I.M. Phase stability and transformation of the $\alpha$ to $\varepsilon$-phase of Alq3 phosphor after thermal treatment and their photo-physical properties. J. Phys. Chem. Solids 2018, 121, 396-408. [CrossRef]

11. Fukushima, T.; Kaji, H. Green- and blue-emitting tris(8-hydroxyquinoline) aluminum(III) (Alq3) crystalline polymorphs: Preparation and application to organic light-emitting diodes. Org. Electron. 2012, 13, 2985-2990. [CrossRef]

12. Pohl, R.; Anzenbacher, P. Emission Color Tuning in AlQ3 Complexes with Extended Conjugated Chromophores. Org. Lett. 2003, 5, 2769-2772. [CrossRef] [PubMed]

13. Pérez-Bolívar, C.; Takizawa, S.Y.; Nishimura, G.; Montes, V.A.; Anzenbacher, P., Jr. High-efficiency tris(8-hydroxyquinoline)aluminum (Alq3) complexes for organic white-light-emitting diodes and solid-state lighting. Chem. Eur. J. 2011, 17, 9076-9082. [CrossRef] [PubMed]

14. Duvenhage, M.M.; Ntwaeaborwa, O.M.; Swart, H.C. UV exposure and photon degradation of Alq3 powders. Phys. B Condens. Matter 2012, 407, 1521-1524. [CrossRef]

15. Kim, K.; Hong, K.; Kim, S.; Lee, J.-L. Doping Mechanism and Electronic Structure of Alkali Metal Doped Tris(8-hydroxyquinoline) Aluminum. J. Phys. Chem. C 2012, 116, 9158-9165. [CrossRef]

16. Bhagat, S.A.; Borghate, S.V.; Kalyani, N.T.; Dhoble, S.J. Novel Na ${ }^{+}$doped Alq3 hybrid materials for organic light-emitting diode (OLED) devices and flat panel displays. Luminescence 2015, 30, 251-256. [CrossRef] [PubMed]

17. Cuba, M.; Muralidharan, G. Enhanced luminescence properties of hybrid Alq3/ZnO (organic/inorganic) composite films. J. Lumin. 2014, 156, 1-7. [CrossRef]

18. Li, J.; Xie, B.; Xia, K.; Zhao, C.; Li, Y.; Hu, S. Enhanced PL and EL properties of Alq3/nano-TiO 2 with the modification of 8-vinyl POSS. Opt. Mater. 2018, 78, 279-284. [CrossRef]

19. Yang, S.J.; Park, C.R. Facile preparation of monodisperse $\mathrm{ZnO}$ quantum dots with high quality photoluminescence characteristics Nanotechnology 2007, 19, 035609. [CrossRef] [PubMed]

20. Iwashita, N. Chapter 2-X-ray Powder Diffraction. In Materials Science and Engineering of Carbon; Inagaki, M., Kang, F., Eds.; Butterworth-Heinemann: Oxford, UK, 2016; pp. 7-25.

21. Mashford, B.; Baldauf, J.; Nguyen, T.-L.; Funston, A.M.; Mulvaney, P. Synthesis of quantum dot doped chalcogenide glasses via sol-gel processing. J. Appl. Phys. 2011, 109, 094305. [CrossRef]

22. Duvenhage, M.-M.; Ntwaeaborwa, M.; Visser, H.G.; Swarts, P.J.; Swarts, J.C.; Swart, H.C. Determination of the optical band gap of Alq3 and its derivatives for the use in two-layer OLEDs. Opt. Mater. 2015, 42, 193-198. [CrossRef] 